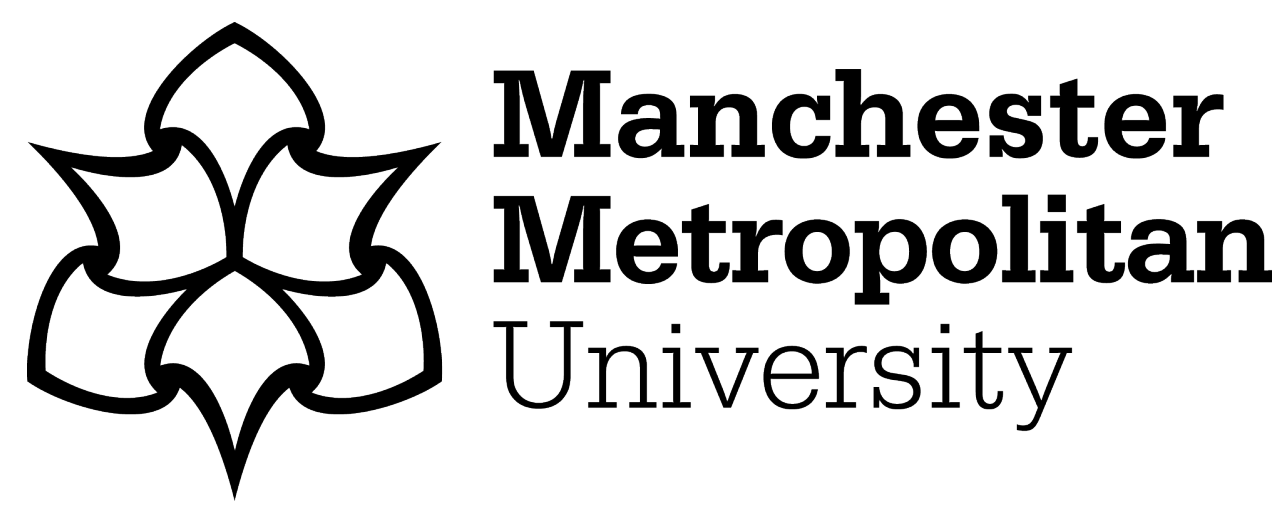

Venkatachalam, Sriram, Agarwal, Shagun, Yan, Shiqiang, Xie, Zhihua, Saincher, Shaswat, Schlurmann, Torsten, Ma, Qingwei, Stoesser, Thorsten, Zhuang, Yuan, Han, Bo, Zhao, Weiwen, Yang, Xiaotong, Li, Z, Wan, Decheng, Zhang, Yi, Teng, Bin, Ning, Dezhi, Zhang, Ningbo, Zheng, Xing, $\mathrm{Xu}$, Guochun, Gong, Jiaye, Li, Yunbo, Liao, Kangping, Duan, Wenyang, Han, Ronggui, Asnim, Windiman, Sulaiman, Zana, Zhou, Zhongbing, Qin, Jianmin, Li, Yucheng, Song, Zhiwei, Lou, Xiaofan, Lu, Lin, Yuan, Changfu, Ma, Yuxiang, Ai, Congfang, Dong, Guohai, Sun, Hanbing, Wang, Qiang, Zhai, Zhi-Tao, Shao, Yan-Lin, Lin, Zaibin, Qian, Ling ORCID logoORCID: https://orcid.org/0000-0002-9716-2342, Bai, Wei, Ma, Zhihua, Higuera, Pablo, Buldakov, Eugeny, Stagonas, Dimitris, Martelo Lopez, Santiago, Christou, Aristos, Lin, Pengzhi, Li, Yanyan, Lu, Jinshu, Hong, Sa Young, Ha, Yoon-Jin, Kim, Kyong-Hwan, Cho, Seok-Kyu, Park, Dong-Min, Laskowski, Wojciech, Eskilsson, Claes, Ricchiuto, Mario, Engsig-Karup, Allan P, Cheng, Lin, Zheng, Jinhai, Gu, Hanbin and Li, Guangnian (2021) A Comparative Study on the Nonlinear Interaction Between a Focusing Wave and Cylinder Using State-of-the-art Solvers: Part A. International Journal of Offshore and Polar Engineering, 31 (1). ISSN 1053-5381

Downloaded from: https://e-space.mmu.ac.uk/627361/

Version: Accepted Version

Publisher: International Society of Offshore and Polar Engineers 
DOI: https://doi.org/10.17736/ijope.2021.jc820

Please cite the published version 


\title{
A comparative study on non-linear interaction between focusing wave and cylinder using state-of-the-art solvers: Part A
}

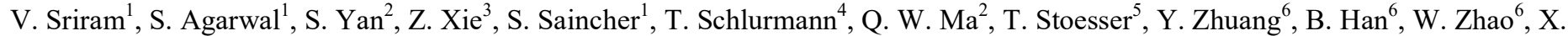

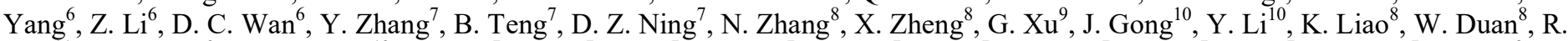

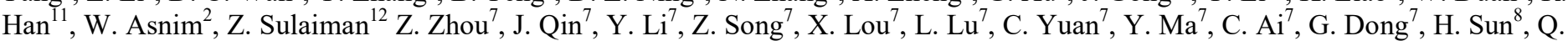 \\ Wang $^{8}$, Z. Zhai ${ }^{8}$, Y. Shao ${ }^{13}$, Z. Lin ${ }^{14}$, L. Qian ${ }^{14}$, W. Bai ${ }^{14}$, Z. Ma ${ }^{14}$, P. Higuera ${ }^{15}$, E. Buldakov ${ }^{5}$, D. Stagonas ${ }^{16,22}$, S. Martelo ${ }^{3}$, A. \\ Christou ${ }^{5}$, P. Lin ${ }^{17}$, Y. Li ${ }^{18}$, J. Lu ${ }^{18}$, S. Y. Hong ${ }^{19}$, Y. J. Ha ${ }^{19}$, K. H. Kim ${ }^{19}$, S. K. Cho ${ }^{19}$, D. M. Park ${ }^{19}$, W. Laskowski ${ }^{13}$, C. Eskilsson ${ }^{20}$, \\ M. Ricchiuto ${ }^{21}$, A. P. Engsig-Karup ${ }^{13}$, L. Cheng ${ }^{23}$, J. Zheng ${ }^{23}, \mathrm{H}^{-\mathrm{Gu}^{9}}, \mathrm{G}^{9} \mathrm{Li}^{9}$ \\ ${ }^{1}$ Indian Institute of Technology Madras, India; ${ }^{2}$ City University of London, UK; ${ }^{3}$ Cardiff University, UK; ${ }^{4}$ Leibniz University of Hannover, \\ Germany; ${ }^{5}$ University College London, UK; ${ }^{6}$ Shanghai Jiao Tong University, China; ${ }^{7}$ Dalian University of Technology, China; ${ }^{8}$ Harbin \\ Engineering University, China; ${ }^{9}$ Ningbo University, China; ${ }^{10}$ Shanghai Maritime University, China; ${ }^{11}$ Yantai CIMC Raffles Offshore Limited, \\ China; ${ }^{12}$ GustoMSC B.V., The Netherlands; ${ }^{13}$ Technical University of Denmark, Denmark; ${ }^{14}$ Manchester Metropolitan University, UK; ${ }^{15}$ The \\ University of Auckland, New Zealand; ${ }^{16}$ Cranfield University, UK; ${ }^{17}$ Sichuan University, China; ${ }^{18}$ Zhajiang Ocean University, China; ${ }^{19}$ Korea \\ Research Inst of Ships \& Ocean Eng, Korea; ${ }^{20}$ Research Institutes of Sweden, Sweden; ${ }^{21}$ Inria Bordeaux-Sud-Ouest, France; ${ }^{22}$ University of \\ Cyprus, Cyprus; ${ }^{23}$ Hohai University, China
}

\section{ABSTRACT}

This paper presents the comparative study carried out for focused waves interaction with the fixed cylinder in ISOPE 2020. The paper discusses qualitative and quantitative comparison between 20 different numerical solvers from various universities across the world for a fixed cylinder. The moving cylinder cases are reported in the companion paper as Part B. The numerical solvers presented in this paper are recent state of the art in the field, mostly developed in-house by various academic institutes. Majority of the participants used hybrid modeling, i.e. a combination of potential flow and Navier-Stokes solvers. The qualitative comparison based on the wave probe and pressure probe time histories and spectral components between laminar, turbulent and potential flow solvers are presented in this paper. Furthermore, the quantitative error analysis based on the overall relative error in peak and phase shift in the wave probe and pressure probe of all the 20 different solvers are reported. The quantitative error with respect to different spectral component energy level (i.e., in primary, sub and super harmonic region) capturing capability are reported. Thus, the paper discusses about maximum, minimum and median relative error present in the recent solvers for application to industrial problems rather than attempting to find the best solver. Further, recommendations are drawn based on the analysis.

KEY WORDS: Comparative study, Hybrid modeling, NavierStokes, Potential flow, Fixed cylinder, Moving Cylinder, Validation.

\section{INTRODUCTION}

Recently, several numerical codes are available as open-source or commercial packages and some are developed in academia. These are based on a variety of underlying mathematical models encompassing both new and traditional computational methods and there still remains considerable uncertainty in their application and reliability. Many attempts are made by the researchers to collaborate and validate their inhouse developed codes (See, Ransley et al., (2019, 2020), Loysel et al., (2012), Clément - ISOPE Benchmark 1 (1999) and Tanizawa and Clément - ISOPE Benchmark 2 (2000)). The performance of these solvers is problem dependent. Whilst a given solver may prove to be the best choice for some applications, it may not perform well for other applications. Hence, these solvers need to be tested for a sufficiently wide range of applications.
In the present study, the laboratory measurements on focusing wave interaction with a fixed cylinder and moving cylinder has been released for the participants to compare with their numerical model. The objective of this comparative study is to (a) understand how well the recent state of the art numerical solvers perform and what type of solvers the participants are preferring for this application (b) instead of finding a best solver, rather estimate overall maximum, minimum and median error that are present in these recent codes (c) assess the overall performance of these solvers in capturing the primary, super harmonics and sub harmonics components, and relative error in pressure and wave probe time history and (d) evaluate the overall performance in simulating small amplitude and large amplitude focusing waves. Finally, a reasonable quantification of the "acceptable error" is provided to the numerical modeling community/users/developers, as a guideline for future investigations; this is something which is currently not available to the best of authors' knowledge. The present paper is an attempt towards meeting these objectives. A similar study 
involving moving cylinder cases is reported in the companion paper as Part B (Agarwal et al., 2021).

\section{EXPERIMENTAL DETAILS}

The experiments were performed using the wave tank in Ludwig-Franzius-Institute at Leibniz University of Hannover, Germany. The experimental results that are used in this paper correspond to the wave characteristics, as shown in Table 1.0, generated based on the second order wavemaker theory. There are seven wave probes and eight pressure transducers deployed to measure the focusing wave evolution and pressures on the cylinder. The details can be referred in Sriram et al., (2021) along with the experimental error analysis in this journal volume and the same shall not be repeated here for the sake of brevity. The constant steepness spectrum has been adopted. The choice of a constant steepness for studying non linearity as opposed to constant amplitude is prudent, since the effects of non-linearity on the evolution of the focusing wave group will be uniform across the spectral components, owing to a single value of steepness across the spectrum. Thus, the numerical model capability can be accessed as the focusing is sensitive to capture the manifestation of the sub and super harmonics components and its spectral evolution correctly.

Table 1.0. Tested wave packet characteristics

$\left(f_{c}\right.$ - center frequency, $\Delta f$ - frequency bandwidth, $t_{f}$ - focusing time, $\mathrm{N}$ - number of wave packets, $\mathrm{G}_{\mathrm{a}}$ - amplitude gain parameter.)

\begin{tabular}{|l|c|c|c|c|c|c|}
\hline Case & $\boldsymbol{f}_{\boldsymbol{c}}(\mathbf{H z})$ & $\Delta \boldsymbol{f} / \boldsymbol{f}_{\boldsymbol{c}}$ & $\boldsymbol{f}_{\boldsymbol{1}} \mathbf{( H z )}$ & $\boldsymbol{t}_{\boldsymbol{f}}(\mathbf{s})$ & $\mathbf{N}$ & $\mathbf{G}_{\mathbf{a}}$ \\
\hline Case 1 & 0.68 & 1.00 & 0.34 & 38.0 & 32 & 0.001 \\
\hline Case 3 & 0.68 & 1.00 & 0.34 & 38.0 & 32 & 0.003 \\
\hline
\end{tabular}

\section{PARTICIPATING CODES AND DATA PROCESSING}

The list of participating universities, the code name/label used in the present papers along with the code details are presented in Table 2.0. There are 20 different codes, each of which is different in terms of the numerical schemes, methodology and physics involved. An interesting observation from this table is that most of the participants used hybrid modeling, i.e. in the far field potential flow theory (referred in Table 2.0 as P) is used and near to the cylinder they have used Navier Stokes (NS) equations, either in the laminar or turbulent frameworks (herein, referred as NSL, NST). In hybrid modeling most of the participants have adopted a weakly coupled approach (or oneway coupling), which is sufficient for the present transient focused wave simulation. The methods involved are Finite Volume (FVM), Finite Element (mostly for potential flow), Finite Difference as well as Particle methods (SPH and MLPGR). Almost all the codes are in-house developed in OpenFOAM with a sole exception being the commercial software STAR-CCM+ employed in a couple of studies. More details about the code methodology and other aspects can be referred in the citations therein or in this volume of the journal. The simulation data from the participants are obtained at a sampling frequency of $100 \mathrm{~Hz}$ for both wave and pressure probe readings at the mentioned locations (See Sriram et al., 2020). Further, it is instructed to the participants to provide the data with respect to wave probe 1 (i.e., incident wave probe at $x=+4.98 \mathrm{~m}$ ), in order to understand the phase shift between the numerical codes. No filtering or noise removal was carried out during the comparison process. Further, some participants have not reported some wave probe or pressure probe results.

\section{RESULTS AND DISCUSSION}

\section{Fidelity of wave-packet generation}

The submitted results have first been evaluated based on how accurately the numerical wavemaker input (either piston-type or a Dirichlet input from the spectrum) develops into wave packets that would undergo focusing near the cylinder. Majority of the participants have used piston type wavemaker, except five codes, wherein Dirichlet type boundary conditions have been used. The participating studies have been grouped into two sets: A and B to facilitate readability of the plots. It may be noted that this segregation is not based on any criteria.

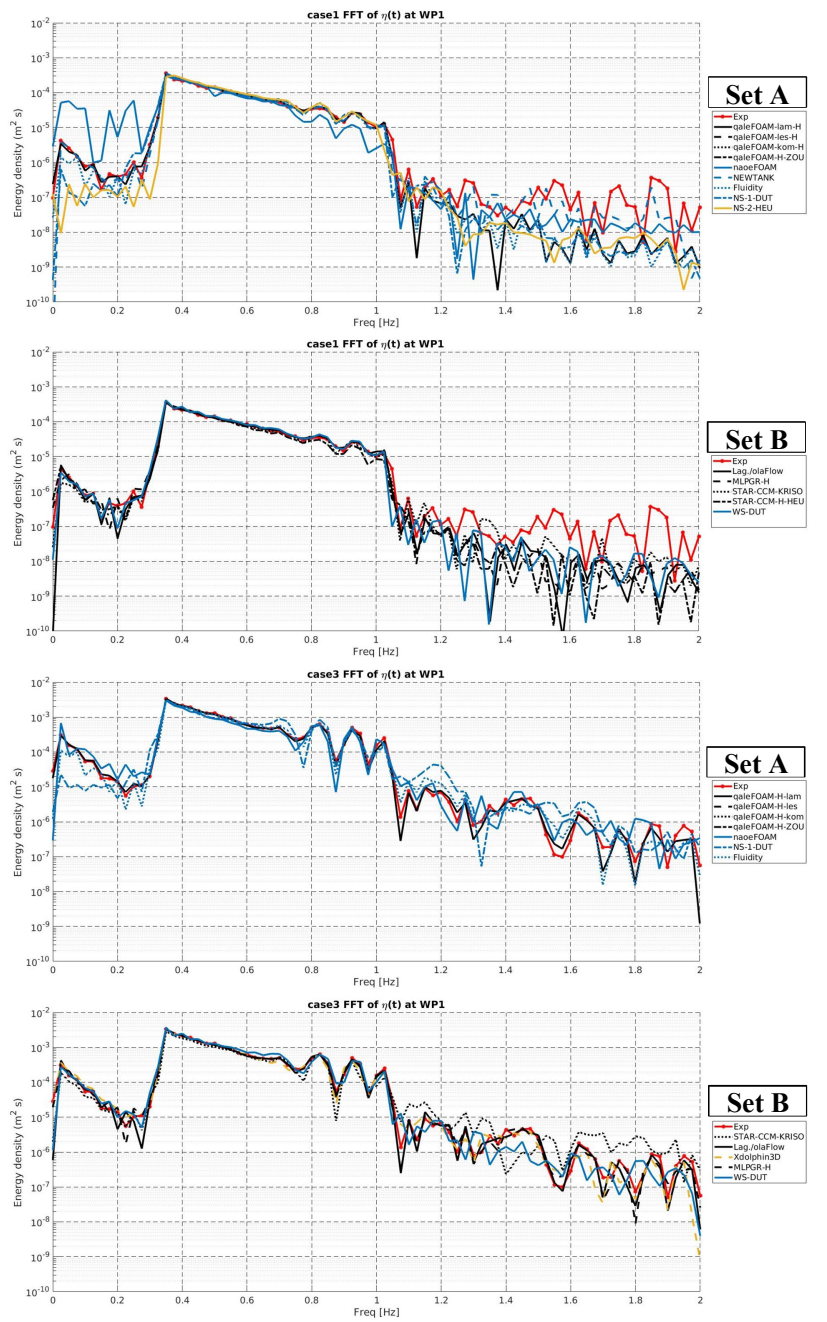

Fig 1. Comparison of spectral energy distribution for surface elevation $\eta(t)$ signals recorded near the wave paddle at WP1 $(x=+4.98 \mathrm{~m})$. 
This shows the advances in implementing the moving boundary in the present modeling technology, which was challenging a decade ago. In order to know the performance, we restrict to a frequency domain analysis as comparison of the input energy at different packet frequencies is of interest. In order to assess the fidelity of wave packet generation, FFT analysis of the freesurface elevation $\eta(t)$ signals recorded by the WP1 gauge close to the wavemaker has been carried out. The results from the comparative analysis are presented in Fig. 1. It may be inferred from Fig. 1 that the general agreement between the simulations and experimental FFT plots is good. In case of the steeper wave (Case 3), the simulations are seen to successfully capture the energy content within the primary range $(f \in[0.34: 1.02 \mathrm{~Hz}])$ as well as for the sub $(0<f<0.34 \mathrm{~Hz})$ and super-harmonics $(1.02<f<2.04 \mathrm{~Hz})$. However, it is also seen from Fig. 1 that a general difficulty exists in capturing the super-harmonic wave packets for the smaller focused wave (Case 1) which seems to be independent of the numerical framework chosen for simulation. Said difficulty may be attributed to the small amplitude of these packets which makes it challenging to capture them in a simulation and numerical damping is inevitable if mesh convergence isn't sufficiently established. Thus, from this study it can be concluded that the capturing of small amplitude focusing is challenging when compared to large amplitude waves, irrespective of the wave generation method employed.

Table 2.0. Details about the participating institutes and numerical codes employed for simulation

(P: Potential; NSL: NS-Laminar; NST: NS-Turbulent; IH: In-house; OS: Open source; C: Commercial; WC: Weak Coupling; $L_{N S}$ : Length of Navier-Stokes sub-domain before cylinder in case of weak-coupling; $D / \Delta x$ : Cells dividing the cylinder diameter where $D$ is the diameter of the cylinder and $\Delta x$ is the minimum horizontal cell size reported by the participants.)

\begin{tabular}{|c|c|c|c|c|c|c|c|c|c|c|}
\hline Sr. & $\begin{array}{l}\text { Name of the } \\
\text { participating } \\
\text { university }\end{array}$ & $\begin{array}{l}\text { Code } \\
\text { Name/Label }\end{array}$ & $\begin{array}{c}\mathrm{P} / \\
\mathrm{NSL} / \\
\mathrm{NST}\end{array}$ & Method & $\begin{array}{l}\text { Turbulence } \\
\text { Scheme }\end{array}$ & $\begin{array}{l}\text { 1-phase } \\
\text { /2-phase }\end{array}$ & $\begin{array}{l}\text { Wavemaker } \\
\text { or source } \\
\text { function }\end{array}$ & $L_{N S}$ & $\frac{D}{\Delta x}$ & $\begin{array}{c}\mathrm{IH} / \\
\mathrm{OS} / \\
\mathrm{C}\end{array}$ \\
\hline 1. & $\begin{array}{l}\text { IIT Madras } \\
\text { Agarwal et al. (2021) }\end{array}$ & $\begin{array}{l}\text { IITM } \\
\text { MLPGR-H }\end{array}$ & $\begin{array}{c}\mathrm{P}- \\
\text { NSL }\end{array}$ & $\begin{array}{c}\text { FEM - } \\
\text { MLPGR }\end{array}$ & -- & 1-phase & Piston $-\mathrm{WC}$ & $4 \mathrm{~m}$ & 6 & $\mathrm{IH}$ \\
\hline 2. & $\begin{array}{l}\text { City, UoL } \\
\text { Li et al. (2018), Yan } \\
\text { et al. }(2020)\end{array}$ & $\begin{array}{l}\text { qaleFOAM- } \\
\text { H-lam }\end{array}$ & $\begin{array}{c}\text { P- } \\
\text { NSL }\end{array}$ & $\begin{array}{l}\text { FEM- } \\
\text { FVM }\end{array}$ & -- & 2-phase & Piston - WC & $\begin{array}{c}2.2 \\
\mathrm{~m}\end{array}$ & 15 & $\begin{array}{l}\mathrm{IH} / \\
\mathrm{OS}\end{array}$ \\
\hline 3. & $\begin{array}{l}\text { City, UoL } \\
\text { Li et al. (2018), Yan } \\
\text { et al. }(2020)\end{array}$ & $\begin{array}{l}\text { qaleFOAM- } \\
\text { H-LES }\end{array}$ & $\begin{array}{c}\text { P- } \\
\text { NST }\end{array}$ & $\begin{array}{l}\text { FEM- } \\
\text { FVM }\end{array}$ & LES & 2-phase & Piston - WC & $\begin{array}{c}2.2 \\
\mathrm{~m}\end{array}$ & 61 & $\begin{array}{l}\mathrm{IH} / \\
\mathrm{OS}\end{array}$ \\
\hline 4. & $\begin{array}{l}\text { City, UoL } \\
\text { Li et al. (2018), Yan } \\
\text { et al. }(2020)\end{array}$ & $\begin{array}{l}\text { qaleFOAM- } \\
\text { H-kom }\end{array}$ & $\begin{array}{c}\text { P- } \\
\text { NST }\end{array}$ & $\begin{array}{l}\text { FEM- } \\
\text { FVM }\end{array}$ & $\mathrm{k}-\omega \mathrm{SST}$ & 2-phase & Piston - WC & $\begin{array}{c}2.2 \\
\mathrm{~m}\end{array}$ & 22 & $\begin{array}{l}\mathrm{IH} / \\
\mathrm{OS}\end{array}$ \\
\hline 5. & $\begin{array}{l}\text { SMU } \\
\text { Li et al. (2018), Yan } \\
\text { et al. }(2020)\end{array}$ & $\begin{array}{l}\text { qaleFOAM- } \\
\text { H-SMU }\end{array}$ & $\begin{array}{c}\text { P- } \\
\text { NST }\end{array}$ & $\begin{array}{l}\text { FEM- } \\
\text { FVM }\end{array}$ & k- $\omega$ SST & 2-phase & Piston - WC & $\begin{array}{c}2.2 \\
\mathrm{~m}\end{array}$ & 550 & $\begin{array}{l}\mathrm{IH} / \\
\mathrm{OS}\end{array}$ \\
\hline 6. & $\begin{array}{l}\text { Cardiff Univ. } \\
\text { Xie and Stoesser } \\
(2020)\end{array}$ & Xdolphin3D & NST & FVM & LES & 2-phase & Piston & NA & 11 & $\mathrm{IH}$ \\
\hline 7. & $\begin{array}{l}\text { SJTU } \\
\text { Shen and Wan (2016) }\end{array}$ & $\begin{array}{l}\text { naoe-FOAM- } \\
\text { SJTU }\end{array}$ & NSL & FVM & - & 2-phase & Piston & NA & 17 & $\begin{array}{l}\mathrm{IH} / \\
\mathrm{OS}\end{array}$ \\
\hline 8. & $\begin{array}{l}\text { DUT } \\
\text { Zhang and Teng } \\
(2019), \text { Zhang et al. } \\
(2020)\end{array}$ & WS-DUT & $\mathrm{P}$ & HOBEM & -- & 1-phase & Dirichlet & NA & 44 & $\mathrm{IH}$ \\
\hline 9. & $\begin{array}{l}\text { DUT } \\
\text { Xie et al. (2017) }\end{array}$ & Fluidity & NSL & FEM & DNS & 1-phase & Piston & NA & 1100 & OS \\
\hline 10. & $\begin{array}{l}\text { UoA, UCL } \\
\text { Buldakov et al. } \\
(2019) ; \text { Higuera } \\
(2020)\end{array}$ & Lag./olaFlow & $\begin{array}{c}\text { P- } \\
\text { NST }\end{array}$ & $\begin{array}{l}\text { FDM- } \\
\text { FVM }\end{array}$ & k- $\omega$ SST & 2-phase & Piston - WC & $\begin{array}{c}1.77 \\
\mathrm{~m}\end{array}$ & 147 & $\begin{array}{l}\mathrm{IH} / \\
\mathrm{OS}\end{array}$ \\
\hline 11. & $\begin{array}{l}\text { DUT } \\
\text { Ai et al. (2011); Ai et } \\
\text { al. (2019) and Ma et } \\
\text { al. (2019) }\end{array}$ & NS-1-DUT & $\begin{array}{l}\text { P or } \\
\text { NST }\end{array}$ & FVM & LES & 1-phase & Dirichlet & NA & NA & $\mathrm{IH}$ \\
\hline 12. & $\begin{array}{l}\text { ZOU } \\
\text { Li et al. (2018), Yan }\end{array}$ & $\begin{array}{l}\text { qaleFOAM- } \\
\text { H-ZOU }\end{array}$ & $\begin{array}{c}\mathrm{P}- \\
\text { NSL }\end{array}$ & $\begin{array}{l}\text { FEM- } \\
\text { FVM }\end{array}$ & -- & 2-phase & Piston - WC & $4 \mathrm{~m}$ & 88 & $\begin{array}{l}\mathrm{IH} / \\
\mathrm{OS}\end{array}$ \\
\hline
\end{tabular}




\begin{tabular}{|c|c|c|c|c|c|c|c|c|c|c|}
\hline & $\begin{array}{l}\text { et al. (2020), Gong et } \\
\text { al. (2020) }\end{array}$ & & & & & & & & & \\
\hline 13. & $\begin{array}{l}\text { HEU } \\
\text { Wang et al. (2020) }\end{array}$ & $\begin{array}{l}\text { STAR CCM- } \\
\text { H-HEU }\end{array}$ & $\begin{array}{c}\text { P- } \\
\text { NST }\end{array}$ & FVM & $\begin{array}{c}\text { Realizable } \\
\mathrm{k}-\varepsilon\end{array}$ & 2-phase & Piston - WC & NA & 55 & $\mathrm{C}$ \\
\hline 14. & $\begin{array}{l}\text { HEU } \\
\text { Liao and } \mathrm{Hu}(2013), \\
\text { Liao et al. (2017) }\end{array}$ & NS-2-HEU & NST & FDM & LES (SGS) & 2-phase & Piston & NA & 55 & $\mathrm{IH}$ \\
\hline 15. & $\begin{array}{l}\text { HEU } \\
\text { Zhang et al. (2018) }\end{array}$ & SPH-H & $\begin{array}{c}\text { P- } \\
\text { NSL }\end{array}$ & $\begin{array}{l}\text { FEM- } \\
\text { SPH }\end{array}$ & -- & 1-phase & Piston & NA & 7 & $\mathrm{IH}$ \\
\hline 16. & $\begin{array}{l}\text { MMU } \\
\text { Lin et al. (2019) }\end{array}$ & FNPF-MMU & $\mathrm{P}$ & FVM & -- & 1-phase & Dirichlet & NA & 40 & $\mathrm{IH}$ \\
\hline 17. & $\begin{array}{l}\text { MMU } \\
\text { Chen et al. (2019) }\end{array}$ & $\begin{array}{l}\text { openFOAM- } \\
\text { MMU }\end{array}$ & NSL & FVM & -- & 2-phase & Dirichlet & NA & 147 & OS \\
\hline 18. & $\begin{array}{l}\text { KRISO } \\
\text { Ha et al. (2019) }\end{array}$ & $\begin{array}{l}\text { STAR-CCM- } \\
\text { KRISO }\end{array}$ & NSL & FVM & -- & 2-phase & Dirichlet & NA & 110 & $\mathrm{C}$ \\
\hline 19. & $\begin{array}{l}\text { TUD } \\
\text { Engsig-Karup et al. } \\
\text { (2016) }\end{array}$ & FNPF-SEM & $\mathrm{P}$ & SEM & -- & 1-phase & Relaxation & NA & NA & $\mathrm{IH}$ \\
\hline 20. & $\begin{array}{l}\text { Hohai Univ. } \\
\text { Lin et al. (2016) }\end{array}$ & NEWTANK & NST & FDM & LES & 2-phase & Piston & NA & 22 & $\mathrm{IH}$ \\
\hline
\end{tabular}

\section{Fidelity of wave focusing}

Next, the submitted results are evaluated in terms of the fidelity/closeness with which the simulations resemble the experiments in replicating the wave focusing event in front of the cylinder, after propagation to a distance of $24.31 \mathrm{~m}$. The qualitative assessment is done both in terms of comparing the profile of the focused wave generated just in front of the cylinder (WP5) as well as the energy contents of its constituent harmonics. The time domain assessment of $\eta(t)$ signals recorded at WP5 for the Case 1 and Case 3 is presented at the top in Fig. 2 and Fig. 3, respectively. It can be observed that most of the solvers correctly replicate the focused wave profile with numerical damping only observed in a couple of cases. It is interesting to note that, for the same wave input, a considerable scatter occurs in wave phase at the focusing point; this is especially true for the smaller wave case 1 . This may be attributed to non-capturing of the higher harmonics at WP1, eventually leading to phase shift across various solvers. However, given the fact that the participants were at liberty to choose the numerical framework for simulation, the submissions are not judged based on phase agreement but rather in terms of the energy contents captured during focusing. The spectral energy density spectra corresponding to the $\eta(t)$ signals recorded at WP5 for the Case 1 and Case 3 are presented at the bottom in Fig. 2 and Fig. 3, respectively. Barring a couple of solvers, almost all models are accurate in predicting the spectral energy within the sub-harmonic and primary frequency bands $(f \leq 1.02 \mathrm{~Hz})$. However, a large discrepancy is observed between the simulations and experiments as well as amongst the different models in capturing the energy contents of the super-harmonics; this is especially noticeable for Case 3 .

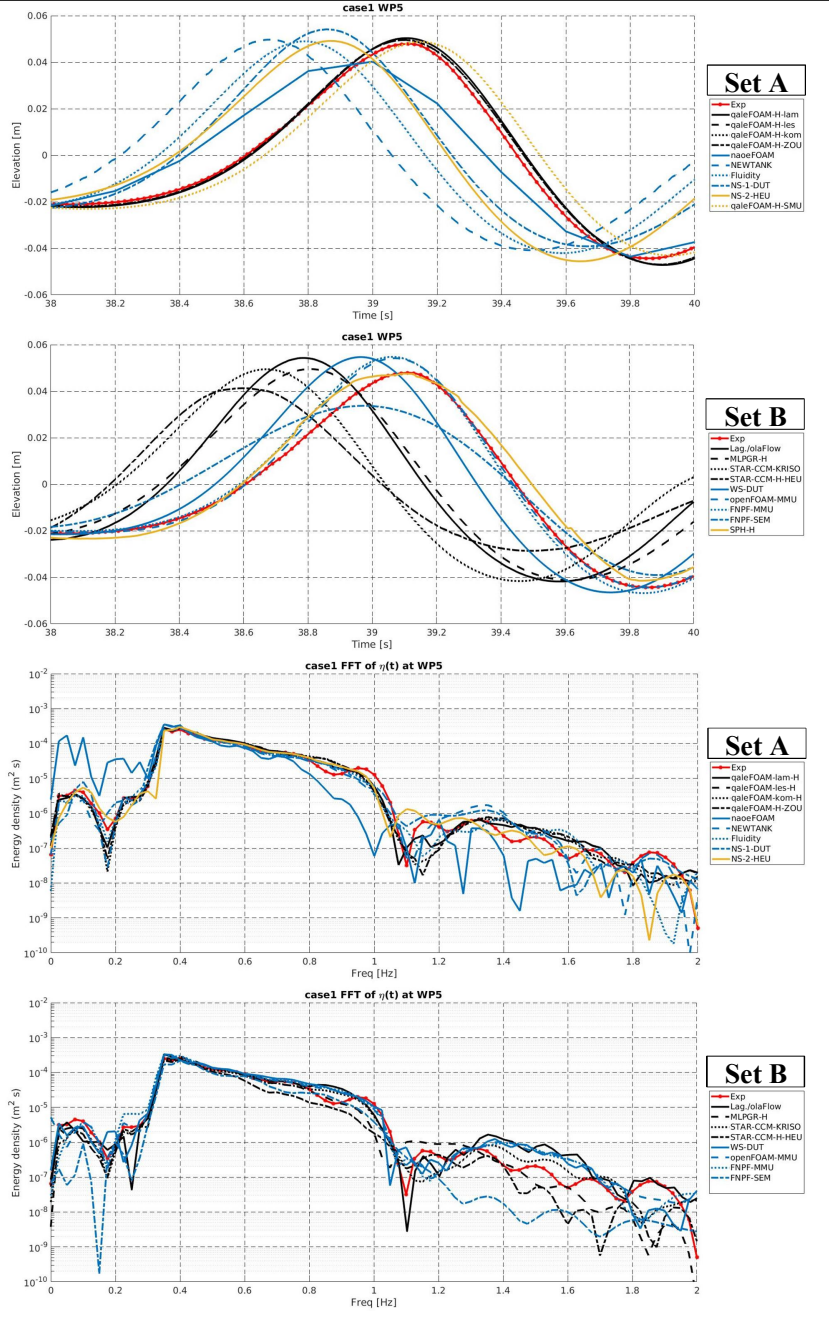

Fig 2. Comparison of time series and spectral energy distribution for $\eta(t)$ signals recorded in front of the cylinder at WP5 $(x=+24.31 \mathrm{~m})$ for the smaller wave (Case 1). 
It is worth mentioning that, owing to the broad-banded nature of the target spectrum, there exists an overlap between the main energy band and the super-harmonics (Sriram et al., 2015) which is visible in the experimental spectra at $f \gtrsim 0.75 \mathrm{~Hz}$.
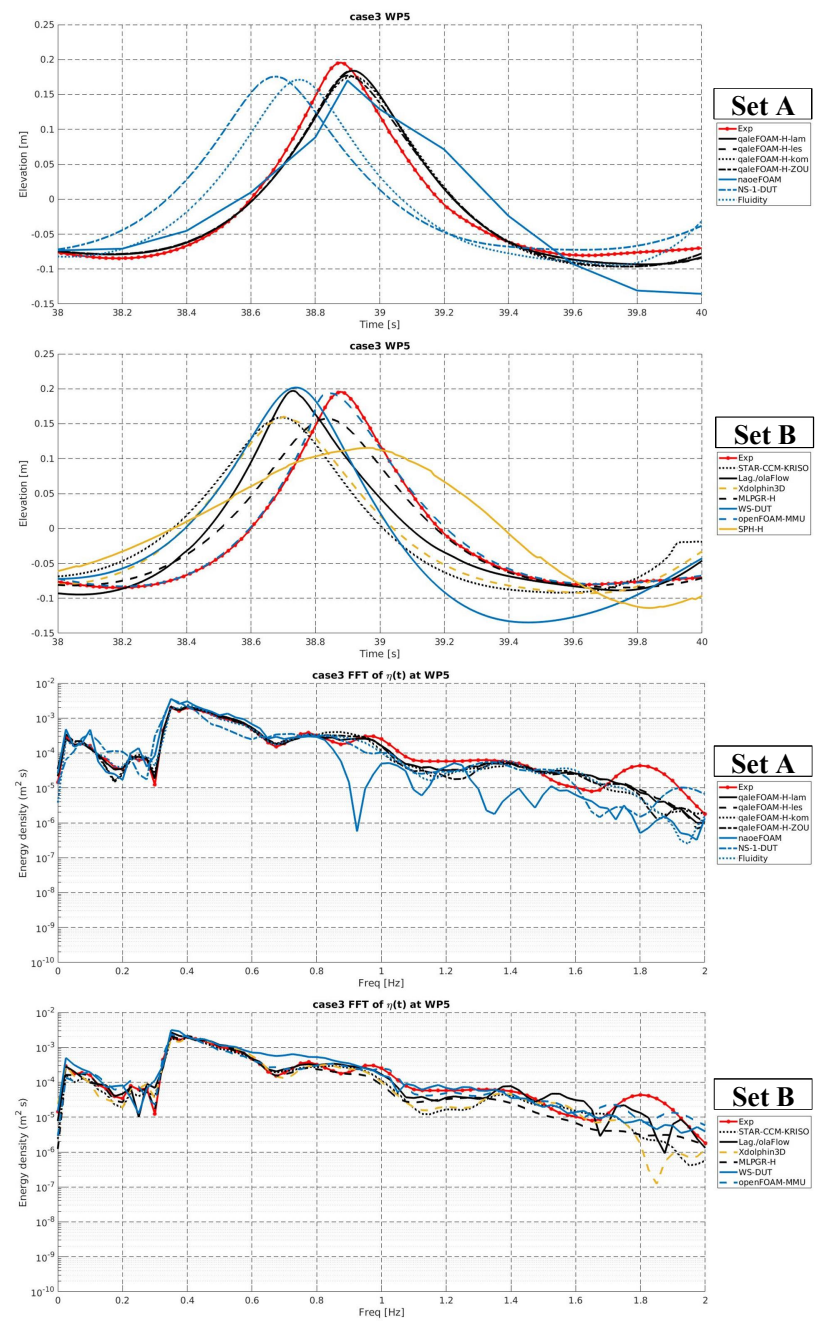

Fig 3. Comparison of time series and spectral energy distribution for $\eta(t)$ signals recorded in front of the cylinder at WP5 $(x=+24.31 \mathrm{~m})$ for the larger wave (Case 3).

It may be noticed that numerical capturing of this overlap is particularly challenging, due to the nonlinear wave-wave interactions as none of the solvers have been able to capture it accurately. It may be concluded from Fig. 2 and 3 that the fidelity of replicating the focusing event is comparable across the various models. Whilst a majority of the solvers either use the full Navier-Stokes equations or hybrid modeling, neither modeling strategy shows any distinctive advantage in replicating the focusing event. It is interesting to note that the pure-FNPT based "WS-DUT" model (set B) achieves excellent phase agreement but tends to over-predict the surface elevation, especially for the case 3, as normally noticed in FNPT.

\section{Accuracy in capturing Pressure}

The ability of the different solvers to replicate the focused wave pressure onto the structure is assessed here by comparing the time variation of dynamic pressure recorded on the submerged and exposed surfaces of the cylinder (see Fig. 4).
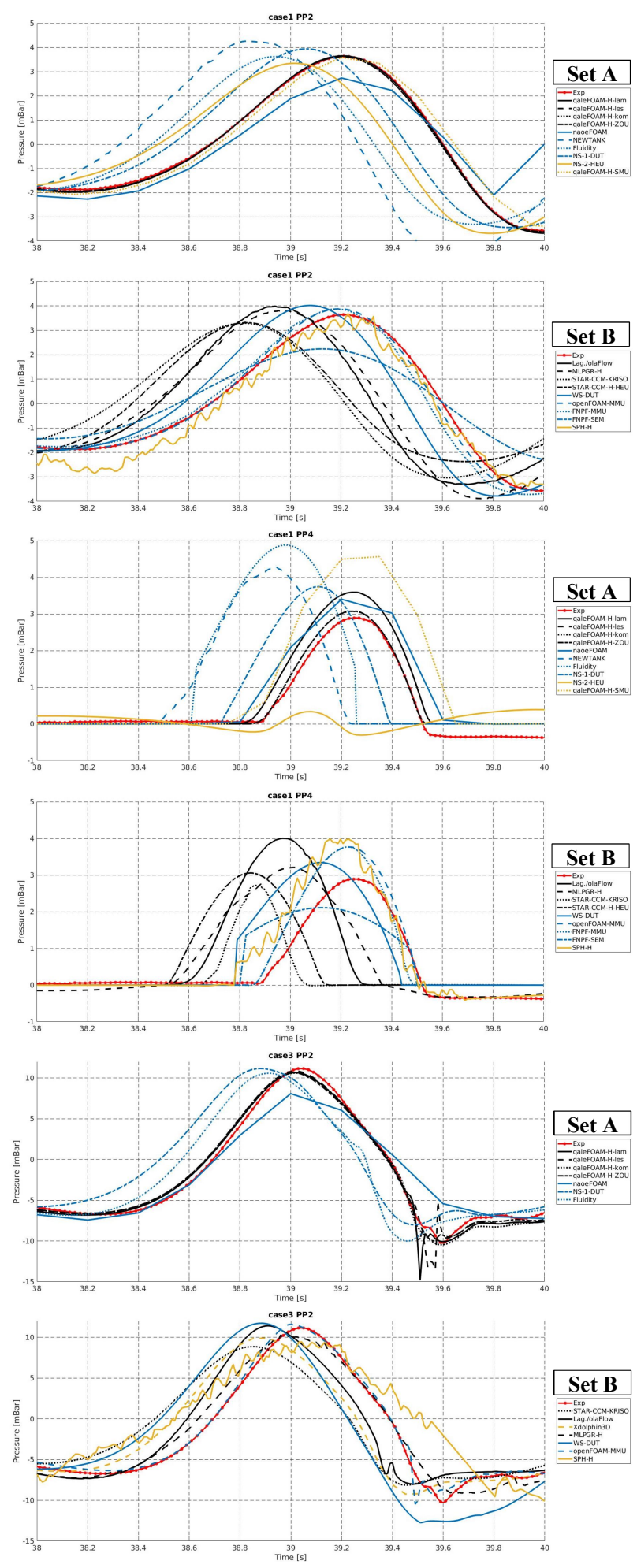


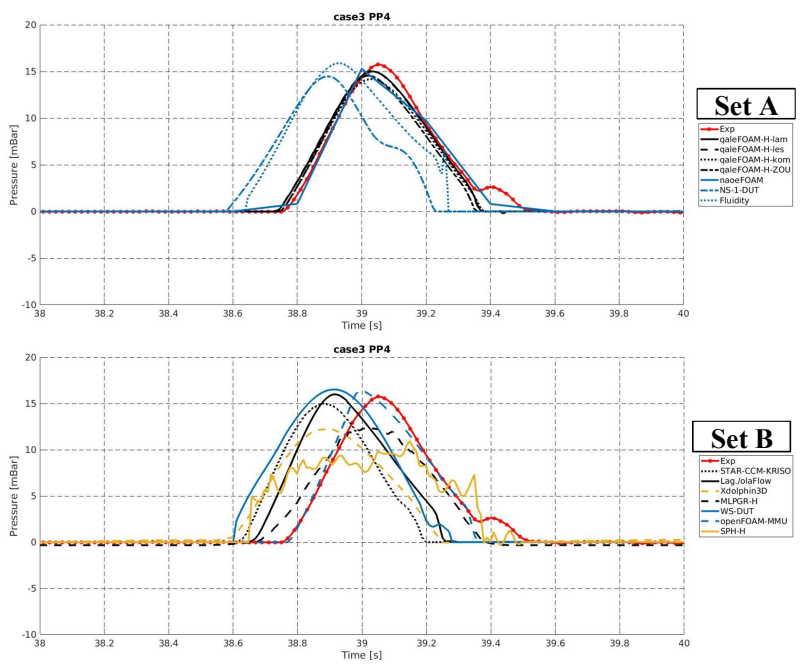

Fig 4. Comparison of time series of dynamic pressure $p(t)$ signals recorded on the surface of the cylinder at probes located below (PP2: $z=-0.185 \mathrm{~m})$ and above the SWL (PP4: $z=+0.015 \mathrm{~m})$.

The evaluation is made for the probes PP2 and PP4 which are placed at $z=-0.185 \mathrm{~m}$ and $z=+0.015 \mathrm{~m}$ from the SWL and oriented at $0^{\circ}$, that is, facing wave attack. Given that PP2 lies at the stagnation point, the dynamic pressure variation is expected to resemble the incident wave elevation (at WP5). Accordingly, the simulated pressure histories from different solvers also exhibit the same trends as previously observed for WP5 in Figs. 2 and 3. That is, the simulations show an overall good agreement against experiments with a greater phase deviation being observed for the smaller wave in comparison to the larger wave. In case of the "air-probe" PP4, the intermittent loading event is correctly reproduced by a majority of the solvers for the steeper wave. However, a significant discrepancy exists in the simulated peak pressure values as well as loading duration for the smaller wave. Given that phase-shift is not a major concern, the fact that the different solvers agree well in predicting the loading induced by the steeper wave is, in fact, promising from the designer's perspective. However, it is to be noted that the uncertainty in numerically predicting the loading induced by the smaller wave is much greater in comparison to that induced by the steeper wave.

\section{Quantification of relative errors}

The qualitative assessment shows the trend which provides an overall understanding of the variations encountered with different solvers. However, one cannot infer any conclusions from the qualitative assessment as only two wave probe and two pressure probe readings have been analyzed. Hence, the relative errors in physical quantities computed through the different solvers for 4 pressure probes and 3 wave probes near the cylinder are analyzed and presented here in terms of quartiles. The box plots for relative error in peak values of pressure $\left(\frac{p_{\max }^{\text {mum }}-p_{\max }^{\text {exp }}}{p_{\max }^{\text {exp }}}\right)$ and surface elevation $\left(\frac{\eta_{\max }^{\operatorname{mum}}-\eta_{\max }^{\text {exp }}}{\eta_{\max }^{\exp }}\right)$, recorded at various probe locations, are shown in Fig. 5. Here, quartiles for NSL and NST models are also reported separately to gain further insight into the capabilities of NS solvers which have been used in $\sim 90 \%$ of the studies (see Table 3.0). If one observes the quartiles of relative error in pressure, it can be seen that all solvers estimate the wave-induced dynamic pressure below the SWL at the stagnation point (PP2) within an error range of $-25 \%$ to $+10 \%$. For the steep wave (Case 3), the tendency of the methods to under-predict the dynamic pressure increases slightly as the median error shifts from $\sim 0 \%$ for the smaller wave to $-5 \%$ for the steeper wave.
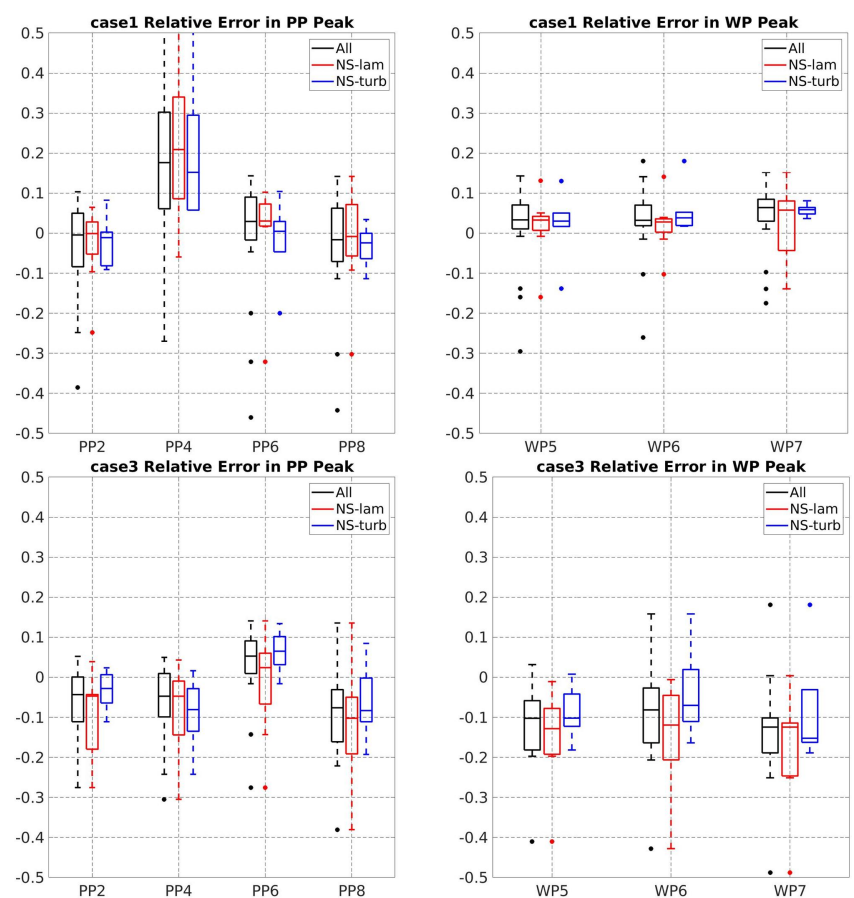

Fig 5. Quartile-based representation of the relative error in peak values of pressure and surface elevation recorded at different probe locations and compared for different groups of methods.

With regards to PP4, which is $0.015 \mathrm{~m}$ above the SWL and thus experiences intermittent loading, the quartiles indicate that the under-prediction in the pressure peak is restricted to $-10 \%$ within the inter-quartile range for the steep wave (Case 3). However, the intermittent pressure peak gets largely overpredicted for the smaller wave such that the median error is almost $+20 \%$. Whilst small waves may not be deemed significant from a structural survivability perspective, the error quartiles indicate that the simulation of intermittent loading induced by small waves is indeed computationally challenging. The probes PP6 and PP8 are submerged at $z=-0.085 \mathrm{~m}$ from the SWL but are offset by $20^{\circ}$ and $180^{\circ}$ from the wave attack. Thus, PP6 lies between the stagnation and separation points whilst PP8 lies adjacent to the cylinder wake. The quartile plots in Fig. 5 indicate that at least $50 \%$ of the solvers (inter-quartile range) are able to predict the dynamic pressure peak at PP6 within an error range of $0-10 \%$ for both Cases 1 and 3 . Strictly speaking in terms of the size of inter-quartile range, this is more or less the same range of uncertainty as previously observed for PP2 from Fig. 5. This should not come as a 
surprise since PP6 lies closer to the stagnation point and thus static pressure variations caused by boundary layer separation (which occur close to $90^{\circ}$ ) are negligible at $20^{\circ}$. Further, this error behavior is seen to be independent of the wave steepness. Nonetheless, the wave steepness is seen to indeed influence the relative error at PP8 where the median of the solvers tends to correctly predict the dynamic pressure peak in the wake for the smaller wave but under-predict the peak for the steep wave. It is also worth noting that the overall relative error range is larger for the steeper wave at PP8. This may be related to stronger wake separation and turbulence effects induced for the steeper wave which lead to an increase in the median error as well as inter-quartile error range for numerical predictions. Overall, the acceptable median error is $\pm 5 \%$ with maximum and minimum acceptable ranges as $\pm 10 \%$. There are few solvers that show extreme values (dots in Fig. 5), those are not acceptable. If one compares the overall performance of all studies against that of the NS solvers, it can be seen that the joint performance is dictated by the relative fidelity of the NSL and NST solvers as the median error of all studies is seen to always lie between the median errors of the NSL and NST studies. This is to be expected since the NS solvers constitute almost $90 \%$ of the submitted results. It is also interesting to note that the inclusion of turbulence modeling invariably results in an improvement in accuracy (shrinking of the interquartile range) in all cases, especially for the sub-surface hydrodynamic loading induced by the steeper wave at the stagnation point (PP2) and in its vicinity (PP6).

The quartiles of relative error in the peak value of free-surface elevation measured at WP5-7 have also been reported on the right side in Fig. 5. It can be appreciated that all submitted solvers captured the focused peak accurately with the median error restricted to $\leq+5 \%$ for the smaller wave at WP5 and WP6. Further, the inclusion of turbulence modeling is seen to improve the surface elevation prediction after the cylinder (WP7), especially for the smaller wave. However, for the steeper wave, there is a tendency for the simulations to underpredict the elevation of the focused peak as the median error is seen to be $\sim-10 \%$ for all the three probes. Further, the inclusion of turbulence modeling is not seen to have any significant impact in improving the prediction of the steeper focused peak as the inter-quartile range as well as the median error remains more or less the same across WP5-7. This tendency to under-predict the focused peak may be closely linked to a corresponding under-prediction of the superharmonic spectral energy content as evidenced from Fig. 3 and will be discussed later. The latter may in-turn be linked to the limitations of the computational mesh in capturing the shorter higher harmonic waves that are generated through nonlinear wave-wave interactions during the focusing process (Sriram et al., 2015). The wave-wave interactions are particularly manifested in the constant steepness approach wherein the steepness is constant throughout the spectral components.

In order to obtain greater physical insight into the processes of focused wave generation and its interaction with the cylinder and how well the same were reproduced in the simulations, the relative energy error $\left(\frac{\sum s(f)^{\text {num }}-\sum s(f)^{\text {exp }}}{\sum S(f)^{\exp }}\right)$ quartiles have been represented over two frequency bands: (a) the primary band containing the focused wave $(f \in[0.34: 1.02 \mathrm{~Hz}])$ and $(\mathrm{b})$ the sub $(0<f<0.34 \mathrm{~Hz})$ and super-harmonics $(1.02<f<$ $2.04 \mathrm{~Hz}$ ) that fall outside the main band. The box plots quantifying relative error in spectral energy content $(S(f)$ ) corresponding to pressure and surface elevation recorded at various probe locations are shown in Fig. 6 for cases 1 and 3.
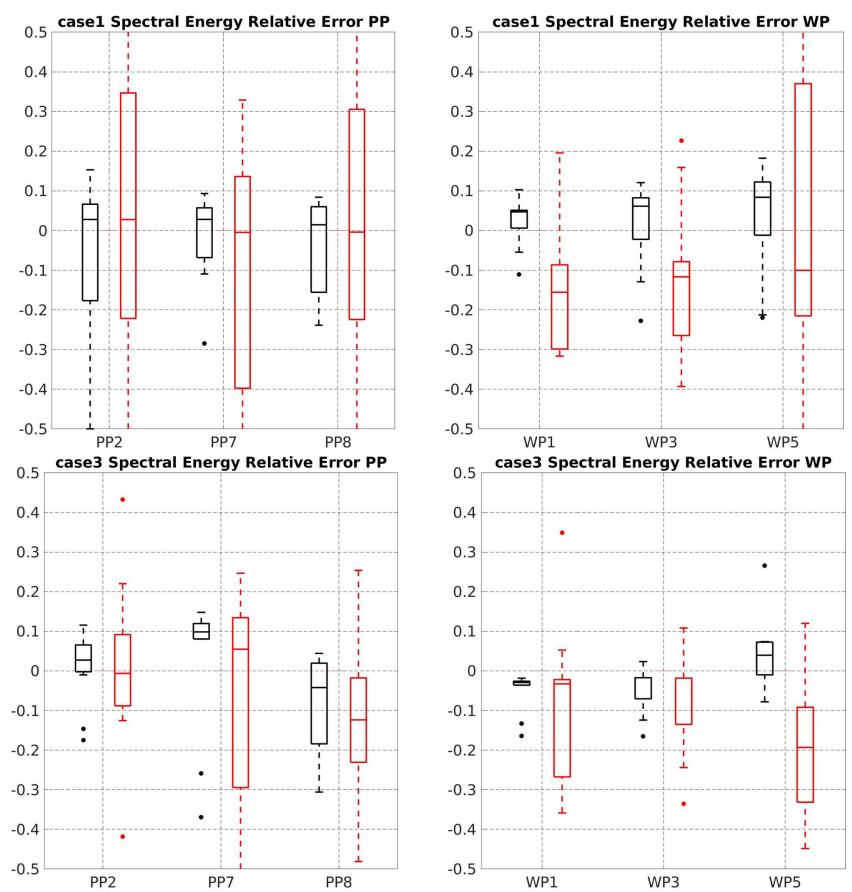

Fig 6. Quartile-based representation of the relative error in spectral energy content within the primary band (black color) as well as sub and super-harmonics (red color) corresponding to $\eta(t)$ and $p(t)$ series recorded at different probe locations.

Referring to Fig. 6, the spectral energy error, corresponding to the pressure signals, has been depicted for the stagnation point (PP2), the separation point (PP7) and the wake (PP8). Broadly speaking, the dynamic pressure corresponding to the main frequency band has been captured more accurately for the steeper wave (Case 3). The greater spread in spectral energy error for the Case 1 may be attributed to a general limitation of the numerical models in not being able to capture the low amplitude variations induced in sub-surface pressure by the smaller waves. This statement is corroborated by the significantly larger error spread in the sub and super-harmonic ranges at PP2 for the Case 1 (in comparison to Case 3) which hints towards a general difficulty in capturing small amplitude, high-frequency waves in the simulations. Interestingly, the relative energy error trend is slightly different in the wake region (PP8). The relative error spread in capturing the wake pressure within the main frequency range is comparable for both waves with the models exhibiting a general tendency to under-predict the wake pressure. However, outside the main range, the median error is $\sim 0 \%$ for the Case 1 . This means that 
the solvers are equally likely to under-predict or over-predict the wake pressure in the sub and super-harmonic ranges for the smaller wave. In contrast, the quartiles indicate that almost $75 \%$ of the solvers would under-predict the sub and superharmonics' induced pressure within wake for the larger wave. Lastly, the relative energy error quartiles for the surface elevation signals are briefly discussed here. A clear-cut trend of the surface elevation error emerges within the main frequency band from Fig. 6. Irrespective of the focused wave design, the error is observed to show an increasing trend (the quartiles spread wider) as one moves from WP1 (close to the wavemaker) to WP3 ( 14 $\mathrm{m}$ from the wavemaker) and finally to WP5 (in front of the cylinder) along the numerical wave tank. This is indicative of a general accumulation of numerical error in the simulations but also suggests that significant effort was put in by the participants towards getting a near-exact match against experiments for the $\eta(t)$ signal at WP1. Obviously, this is to be expected since the free-surface elevation history at WP1 represents the "incident wave packets" whose fidelity is crucial to the accuracy of the entire simulation. It is also noticed from Fig. 6 that the relative energy error for the surface elevation is significantly greater outside the main frequency range. While this is true for both waves, the quartiles spread wider for Case 1, which yet again highlights an inherent limitation of the computational solvers to sufficiently capture the sub (insufficient cells/particles to resolve waveheight) and super-harmonics (insufficient cells/particles to resolve wavelength) of the smaller wave. Further, the quartiles indicate a general increase in relative spectral energy error outside the main band as one move from WP1 to WP5 with the Table 3.0. Computational aspects of the different solvers energy getting under-predicted in case of the steep wave. This, yet again, is attributable to accumulation of numerical error and/or numerical damping of the waves, especially after $\sim 25 \mathrm{~m}$ of propagation to reach WP5 close to the cylinder.

\section{Comparison of CPU effort}

One may presume that the current advancement in computational power, hardware capacity, high performance computing and software architecture would lead to faster computation of nonlinear focused wave interactions with a fixed cylinder in a large domain. Somewhat contrary to this presumption, Table 3.0 indicates that the run-time for a single simulation is more than a day in most of the codes and these simulations have been carried out mostly using a workstation. In particular, most of the in-house codes are OpenMP or MPI paralleled. It is also worth noting from Table 3.0 that the hybrid methodologies are about $2 \times$ faster in comparison to the full/conventional NS solvers and yet achieve a comparable level of accuracy. However, the simulation time for some NST (LES) computation using a hybrid method is still $\sim 2$ days which is significant. This shows that there still exists scope for improvement in solver capability to speed up the computations for large-scale industrial needs. However, a need to develop new methodology should be considered in these directions, such as matrix free algorithms and dynamic programming, rather than relying upon augmentation in high performance computing architecture.

\begin{tabular}{|c|c|c|c|c|c|c|c|c|}
\hline S.N. & Code Name & Processor model & $\begin{array}{l}\text { OpenMP, } \\
\text { MPI, GPU, } \\
\text { Serial }\end{array}$ & $\begin{array}{l}\text { No. of } \\
\text { CPUs }\end{array}$ & $\begin{array}{c}\text { Processor } \\
\text { clock-speed } \\
(\mathrm{GHz})\end{array}$ & $\begin{array}{l}\text { System } \\
\text { RAM } \\
\text { (GB) }\end{array}$ & $\begin{array}{c}\text { Wall- } \\
\text { clock run- } \\
\text { time (hrs) }\end{array}$ & $\begin{array}{c}\text { System } \\
\text { run-time } \\
(\mathrm{hrs})\end{array}$ \\
\hline 1. & IITM MLPGR-H & $\begin{array}{l}\text { Intel(R) Xeon(R) } \\
\text { CPU E5-2650 v4 }\end{array}$ & $\begin{array}{c}\text { Serial/ } \\
\text { OpenMP }\end{array}$ & 8 & 2.2 & 34 & 11.9 & -- \\
\hline 2. & $\begin{array}{l}\text { qaleFOAM-H- } \\
\text { lam }\end{array}$ & $\begin{array}{l}\text { Intel(R) Xeon(R) } \\
\text { CPU E5-2680 v4 }\end{array}$ & $\begin{array}{l}\text { OpenMP } \\
\text { MPI }\end{array}$ & 8 & 2.4 & 64 & 7.98 & -- \\
\hline 3. & $\begin{array}{l}\text { qaleFOAM-H- } \\
\text { LES }\end{array}$ & $\begin{array}{l}\text { Intel(R) Xeon(R) } \\
\text { CPU E5-2680 v4 }\end{array}$ & $\begin{array}{l}\text { OpenMP } \\
\text { MPI }\end{array}$ & 16 & 2.4 & 64 & 42 & -- \\
\hline 4. & $\begin{array}{l}\text { qaleFOAM-H- } \\
\text { kom }\end{array}$ & $\begin{array}{l}\text { Intel(R) Xeon(R) } \\
\text { CPU E5-2680 v4 }\end{array}$ & $\begin{array}{l}\text { OpenMP } \\
\text { MPI }\end{array}$ & 16 & 2.4 & 64 & 11.5 & -- \\
\hline 5. & $\begin{array}{l}\text { qaleFOAM-H- } \\
\text { SMU }\end{array}$ & $\begin{array}{l}\text { Intel(R) Xeon(R) E5 } \\
2690 \mathrm{v} 3\end{array}$ & MPI & 7 & 2.4 & 32 & -- & -- \\
\hline 6. & Xdolphin3D & $\begin{array}{l}\text { Intel(R) Xeon(R) } \\
\text { CPU E5-2660 v4 }\end{array}$ & Serial/MPI & 1 & 2.0 & 256 & 36 & -- \\
\hline 7. & $\begin{array}{l}\text { naoe-FOAM- } \\
\text { SJTU }\end{array}$ & $\begin{array}{l}\text { Intel(R) Core(TM) } \\
\text { i7-7700 }\end{array}$ & OpenMP & 6 & 3.6 & 32 & 79.3 & 78.9 \\
\hline 8. & WS-DUT & $\begin{array}{l}\text { Intel(R) Core(TM) } \\
\text { i7-8700K }\end{array}$ & Serial & 6 & 3.7 & 16 & 11.8 & -- \\
\hline 9. & Fluidity & -- & MPI & 56 & -- & -- & 96 & 96 \\
\hline 10. & Lag./olaFlow & $\begin{array}{l}\text { Intel(R) Xeon(R) } \\
\text { Gold } 6138\end{array}$ & Serial/MPI & $1 / 72$ & 2.0 & 64 & $20+35$ & 55 \\
\hline 11. & NS-1-DUT & $\begin{array}{l}\text { Intel(R) Core(TM) } \\
\text { i7-9700K }\end{array}$ & MPI & 8 & 3.6 & 32 & 6.2 & 6.2 \\
\hline
\end{tabular}




\begin{tabular}{|l|l|l|c|c|c|c|c|c|}
\hline 12. & $\begin{array}{l}\text { qaleFOAM-H- } \\
\text { ZOU }\end{array}$ & $\begin{array}{l}\text { Intel(R) Xeon(R) } \\
\text { CPU E5-2680 v4 }\end{array}$ & $\begin{array}{c}\text { OpenMP } \\
\text { MPI }\end{array}$ & 16 & 2.4 & 64 & 21 & -- \\
\hline 13. & $\begin{array}{l}\text { STAR CCM-H- } \\
\text { HEU }\end{array}$ & $\begin{array}{l}\text { Intel(R) Xeon(R) } \\
\text { CPU E5-2080 v2 }\end{array}$ & MPI & 40 & 2.8 & 64 & 62 & -- \\
\hline 14. & NS-2-HEU & $\begin{array}{l}\text { Intel(R) Core (TM) } \\
\text { i7-7700HQ CPU }\end{array}$ & Serial & 4 & 2.8 & 16 & 71.3 & -- \\
\hline 15. & SPH-H & Intel(R) i7 & OpenMP & 16 & 3.3 & 128 & -- & 4.44 \\
\hline 16. & FNPF-MMU & $\begin{array}{l}\text { Intel(R) Xeon(R) } \\
\text { E5-2600 }\end{array}$ & MPI & 16 & 1.7 & 64 & 10.23 & 10.21 \\
\hline 17. & $\begin{array}{l}\text { openFOAM- } \\
\text { MMU }\end{array}$ & $\begin{array}{l}\text { Intel(R) Xeon(R) } \\
\text { E5-2600 }\end{array}$ & MPI & 96 & 1.7 & 64 & 31.16 & 31.09 \\
\hline 18. & $\begin{array}{l}\text { STAR-CCM- } \\
\text { KRISO }\end{array}$ & $\begin{array}{l}\text { Intel(R) Xeon(R) } \\
\text { CPU E5-2640 v4 }\end{array}$ & MPI & 300 & 2.4 & 192 & -- & $\sim 6$ \\
\hline 19. & FNPF-SEM & $\begin{array}{l}\text { Intel(R) Xeon(R) } \\
2660 v 3\end{array}$ & Serial & 6 & 2.6 & 16 & 96 & -- \\
\hline 20. & NEWTANK & $\begin{array}{l}\text { Intel(R) Xeon(R) } \\
\text { CPU E5-2650 }\end{array}$ & OpenMP & 2 & 2.3 & 64 & 96 & 96 \\
\hline
\end{tabular}

\section{CONCLUSIONS}

The paper discusses the comparative study of 20 different solvers, as part of the experimental data released in ISOPE 2020. Based on the analysis carried out in the paper, the following observations are provided which are applicable to a given solver for focused wave-monopile interactions:

(1) Near to the wavemaker $(<5 \mathrm{~m})$, the performance of all the solvers is promising. Thus, the acceptable relative error for a solver in capturing the primary energy content should be $\leq 5 \%$, irrespective of the method of wave-generation adopted.

(2) Far away from the wavemaker (say $\sim 25 \mathrm{~m}$, i.e., $10 \times$ the average wavelength), the relative error increases to $\sim 10 \%$ in primary energy content. However, higher deviations $(\sim 50 \%)$ are expected for sub and superharmonic components manifested through nonlinear wave-wave interactions during propagation.

(3) The error, in general, will be higher for a small amplitude case compared to a steep case in focusing waves. However, in order to ensure sufficient accuracy of the simulation, the error should be restricted within the limits specified in guidelines (1) and (2) in terms of the energy content. In the present comparative study, all the solvers captured the focused wave peaks within a median relative error $\sim+5 \%$ for the smaller wave and $\sim-10 \%$ for the steep wave, which is promising.

(4) The pressure peaks induced by the focusing event should be captured within an error range of $\pm 5 \%$. All the solvers in the present study captured the pressure peaks with a median relative error of $< \pm 4 \%$.

(5) The deviations in phase shift may be attributed to the deviations in sub and super harmonics. However, performance of a given solver in context to industrial applications need not be judged based on phase-shift as the design focus would be on accurately estimating the values of peak loads/pressures.
(6) The "pure" FNPT simulations tend to over-predict the surface elevation as well as the wave-induced pressure at the point of focusing but achieve a nearexact agreement in phase with the experiments.

(7) The inclusion of a turbulence model invariably improves accuracy especially in the prediction of sub-surface pressure induced by steep waves in the vicinity of the forward stagnation point.

(8) Further, method of generations using piston, relaxation zone, Dirichlet type are all within acceptable limit and one is not superior to others.

Thus, based on the results obtained from 20 different solvers, the above observations have been noticed which may be followed. It should be noted that the experimental error/uncertainty should also be taken into consideration during validation (see Sriram et al., 2020). Inclusion of the experimental uncertainty would make the above guidelines less stringent; however it is always better to be conservative (and maintain a reduced error margin) when adopting the said guidelines in practice. In case of deviations, the solver would have to be improved for wave interactions with fixed structure. It is also worth mentioning that the above guidelines hold irrespective of regular or random waves, as the tested conditions are for small amplitude and steep focused waves generated using a constant steepness spectrum. Nevertheless, experiments involving regular and steep breaking focused waves interacting with a fixed as well as moving cylinder may also be considered within the scope for future comparative studies. Further, the state of the art in modeling large domain problems for transient waves appears to be based on hybrid numerical modeling using weakly coupled algorithms (or one way coupling); this strategy was adopted by most of the participants. For this coupling, the length of the domain used by many authors (i.e. from inlet to cylinder location) varies between $2 \mathrm{~m}$ and $4 \mathrm{~m}$. However, it is noteworthy that strongly coupled algorithms have not been adopted by any of the researchers in this comparative study probably because the same are preferred only for non-transient periodic waves. A 
strong coupling between constituent solvers should be adopted in the future (even though challenging to implement) for reflection dominant cases. The present comparative study is carried out for small scale experimental studies; the future comparative direction should also be based on large scale study including air entrainment and compressibility effects.

\section{ACKNOWLEDGEMENTS}

The first author would like to thank Alexander Von Humboldt Foundations and DAAD for the experimental measurements carried out at LuFI, University of Hannover, German, as well as DSTUKIERI project (2016-17-0029 \& DST/INT/UK/P-122/2016).

\section{REFERENCES}

Agarwal, S, et al. (2021). "A comparative study on non-linear interaction between focusing wave and moving cylinder using state-of-the-art solvers: Part B," Int. J. Offshore Polar Eng. Under review.

Ai, C, Jin, S, and Lv, B (2011). "A new fully non-hydrostatic 3D free surface flow model for water wave motions," Int. J. Numer. Methods Fluids, 66(11), 1354-1370.

Ai, C, Ma, Y, Yuan, C, and Dong, G (2019). "Development and assessment of semi-implicit non-hydrostatic models for surface water waves," Ocean. Modell., 144, 1-15.

Agarwal S., Sriram V., Yan S., Murali K., (2021). "Improvements in MLPG formulation for 3D wave-fixed structure interaction," Comput. Fluids. https://doi.org/10.1016/j.compfluid.2020.104826.

Buldakov, E, Higuera, P, and Stagonas, D (2019). "Numerical models for evolution of extreme wave groups," Appl. Ocean Res., 89, 128 140.

Chen, H, Qian, L, Ma, Z, Bai, W, Li, Y, Causon, DM, and Mingham, CG (2019). "Application of an overset mesh based numerical wave tank for modelling realistic free-surface hydrodynamic problems", Ocean Eng., 176, pp.97-117.

Clément, AH (1999). "Benchmark Test Cases For Numerical Wave Absorption: 1st Workshop of ISOPE Numerical Wave Tank Group, Montréal, May 1998," Proc 9th Int Offshore and Polar Eng Conf, Brest, ISOPE.

Engsig-Karup, AP, Eskilsson, C, and Bigoni, D (2016) "A Stabilised Nodal Spectral Element Method for Fully Nonlinear Water Waves," J. Comput. Phys., 318, pp. 1-21.

Gong, J, Yan, S, Ma, QW, Li, Y (2020) "Added resistance and seakeeping performance of trimarans in oblique waves," Ocean Eng., 216, 107721.

Ha, Y., J, Nam, B., W., Kim, K., H., Hong, S., Y. (2019). “CFD Simulations of Wave Impact Loads on a Truncated Circular Cylinder by Breaking Waves," Int. J. Offshore Polar. Eng., 29 (3), 306-314.

Higuera, P (2020). "Enhancing active wave absorption in RANS models," Appl. Ocean Res., 94, 102000.

Li, Q, Wang, J, Yan, S, Gong, J, and Ma, QW (2018) "A zonal hybrid approach coupling FNPT with OpenFOAM for modelling wavestructure interactions with action of current," Ocean Syst. Eng., 8(4), 381-407.

Lin, Z, Qian, L, Bai, W, Ma, Z, Chen, H, and Zhou, J (2019). "Development of a 3D fully nonlinear potential flow wave tank in framework of OpenFOAM," Proceedings of the ASME $201938^{\text {th }}$ International Conference on Ocean, Offshore and Arctic Engineering, June, 2019, Glasgow, Scotland.

Liao, KP and Hu, CH (2013). "A coupled FDM-FEM method for free surface flow interaction with thin elastic plate," J. Mar. Sci. Technol., 18,1-11.

Liao, KP, Duan, WY, Ma, QW, Ma, S, Zhao, BB, and $\mathrm{Hu}, \mathrm{CH}$ (2017). "Numerical analysis of wave impact loads on semisubmersible platform," Proceedings of the 36th International Conference on Ocean, Offshore and Arctic Engineering, Trondheim, Norway.

Lin, P, Cheng, L, and Liu, D (2016). "A two-phase flow model for wave-structure interaction using a virtual boundary force method," Comput. Fluids, 129, 101-110.

Loysel, T, et al. (2012). "Results of the First Sloshing Model Test Benchmark," Proc 22nd Int Offshore and Polar Eng Conf, Rhodes, ISOPE.

Ma, Y, Yuan, C, Ai, C, and Dong, G (2019). "Comparison between a non-hydrostatic model and OpenFOAM for 2D wave-structure interactions," Ocean Eng., 183, 419-425.

Ransley, E, et al. (2019). "A Blind Comparative Study of Focused Wave Interactions with a Fixed FPSO-like Structure (CCP-WSI Blind Test Series 1)," Int. J. Offshore Polar Eng., ISOPE, 29(2), 113-127.

Ransley, E, et al. (2020). "A Blind Comparative Study of Focused Wave Interactions with Floating Structures (CCP-WSI Blind Test Series 3)," Int. J. Offshore Polar Eng., ISOPE, 30(1), 1-10.

Shen, Z., Wan, D.C. (2016). "An irregular wave generating approach based on naoe-FOAM-SJTU solver." China Ocean Eng., 30, 177192

Sriram, V, Schlurmann, T, and Schimmels, S (2015). "Focused wave evolution using linear and second order wavemaker theory," Appl. Ocean Res., 53, 279-296.

Sriram, V, Agarwal, S, and Schlurmann, T (2021). "Laboratory study on steep wave interaction with fixed and moving cylinder," Int. $J$ Offshore Polar. Eng. Revision submitted.

Tanizawa, K, and Clément, AH (2000). "Report of the 2nd Workshop of ISOPE Numerical Wave Tank Group: Benchmark Test Cases of Radiation Problem, (Brest, May 1999)," Proc 10th Int Offshore and Polar Eng Conf, Seattle, ISOPE.

Wang, Q, Zhai, Z, Liao, K, Ma, Q, and Shao, Y (2020). "Study on Interaction of Steep Focused Waves with Fixed Cylinder Based on CFD Method," Proc 30th Int Offshore and Polar Eng Conf, Shanghai, ISOPE.

Yan, S, Wang, J, Wang, J, Ma, Q and Xie, Z (2020). "CCP-WSI Blind Test using qaleFOAM with an Improved Passive Wave Absorber", Int. J. Offshore Polar Eng., 30(1), 43-52.

Xie, Z, Lu, L, Stoesser, T, Lin, JG, Pavlidis, D, Salinas, P, and Matar, OK (2017). "Numerical simulation of three-dimensional breaking waves and its interaction with a vertical circular cylinder," $J$. Hydrodyn., 29(5), 800-804.

Xie, Z, and Stoesser, T (2020). "A three-dimensional Cartesian cutcell/volume-of-fluid method for two-phase flows with moving bodies," J. Comput. Physics, 416, 109536.

Zhang, Y, and Teng, B (2019). "A Nonlinear Potential Flow Model for High-frequency Wave Loads and Ringing Response of Offshore Wind Turbines," Proc 29th Int Offshore and Polar Eng Conf, Honolulu, Hawaii, USA, ISOPE, 1, 304-311.

Zhang, Y, Teng, B, and Ning, DZ (2020). "Comparative Study on Interaction of Steep Focused Waves with a Fixed Cylinder by HOBEM," Proc 30th Int Offshore and Polar Eng Conf, Shanghai, China, ISOPE, 3, 2228-2235.

Zhang, N.B., Zheng, X, Ma, Q.W., et al. (2018). "A hybrid stabilization technique for simulating water wave-structure interaction by incompressible smoothed particle hydrodynamics (ISPH) method." J. Hydro-Environ. Res., 18, 77-94. 